\title{
Matrice extracellulaire et morphogenèse embryonnaire : rôle de la fibronectine dans les migrations cellulaires
}

\author{
JL Duband \\ ENS, laboratoire de physiopathologie du développement du CNRS, \\ 46, rue d'Ulm, 75230 Paris, Cedex 05, France \\ (15 réunion du groupe Développement, INRA, Paris, 24-26 mai 1989)
}

\begin{abstract}
Résumé - Chez les eucaryotes, une grande partie de la morphogenèse est sous le contrôle de quelques familles de glycoprotéines de surface responsables des interactions des cellules avec leurs voisines et avec la matrice extracellulaire environnante. Dans cette synthèse, nous nous limitons à l'étude du rôle de la fibronectine, une glycoprotéine des espaces extracellulaires, et de son récepteur intégrine, dans les processus de migration d'une population cellulaire particulière, les crêtes neurales, au cours de l'embryogenése précoce. Il apparaît que dans l'embryon, les cellules des crêtes neurales en migration sont associées à de la fibronectine; et dans de nombreux cas, celle-ci disparaît de l'environnement des cellules quand cesse leur déplacement. La fibronectine reconnaît son récepteur intégrine principalement par le biais d'une courte séquence de 4 acides aminés (Arg-Gly-Asp-Ser). Si l'on empêche cette association in vivo ou in vitro, par des anticorps ou des peptides Arg-Gly-Asp-Ser solubles en excès, on perturbe gravement la capacité de migration des cellules des crêtes neurales. Une interaction directe entre la fibronectine et son récepteur à la surface cellulaire est donc nécessaire au déplacement des cellules des crêtes neurales. Cependant, la fibronectine elle-même ne suffit pas à induire le mouvement cellulaire. Les cellules des crêtes neurales ont la capacité intrinsèque de se mouvoir, qui se reflète dans l'organisation des récepteurs intégrines à leur surface, et dans celles du cytosquelette dans le cytoplasme sous-jacent. Une telle organisation permettrait en effet à la cellule de se déformer rapidement et d'exercer une interaction transitoire avec son substrat. Récemment, on a pu mettre en évidence l'existence de plusieurs formes de fibronectine qui diffèrent par plusieurs séquences intramoléculaires. Alors que la ionction de certaines d'entre elles est encore inconnue, d'autres contiennent des domaines de liaison pour des récepteurs dont certains sont des intégrines. Nous avons pu montrer que le déplacement des cellules des crêtes neurales requiert une interaction avec ces séquences. On peut donc imaginer que ces formes de fibronectines ont une répartition restreinte dans l'embryon, ce qui permettrait aux cellules de s'orienter dans celui-ci. Ainsi, des molécules des espaces extracellulaires n'auraient plus seulement un rôle permissif dans le comportement cellulaire, mais pourraient aussi avoir un rôle instructif.
\end{abstract}

morphogenèse / matrice extracellulaire / fibronectine / migration cellulaire / crête neurale

Summary - Extracellular matrix and morphogenesis: role of flbronectin in cell migrations. The neural crest provides a useful paradigm for cell migration and modulations in cell adhesion during morphogenesis. In the present review, we describe the major findings on the role of the extracellular matrix glycoprotein fibronectin and its corresponding integrin receptor in the locomotory behavior of neural crest cells. In vivo, fibronectin is associated with the migratory routes of neural crest cells and, in some cases, it disappears from the environment of the cells as they stop migrating. In 
vitro, neural crest cells show a great preference for fibronectin substrates as compared to other matrix molecules. Both in vivo and in vitro, neural crest cell migration can be specifically inhibited by antibodies or peptides that interfere with the binding of fibronectin to its integrin receptor. However, the migratory behavior of neural crest cells cannot result solely from the interaction with fibronectin. Thus, neural crest cells exhibit a particular organization of integrin receptors on their surface and develop a cytoskeletal network which differs from that of non-motile cells. These properties are supposed to permit rapid changes in the shape of cells and to favor a transient adhesion to the substratum. Recent findings have established that different forms of fibronectin may occur, which differ by short sequences along the molecule. The functions of most of these sequences are not known, except for 1 of them which carries a binding site for integrin receptors. We have demonstrated that this site is recognized by neural crest cells and plays a crucial role in their displacement. It is therefore possible that the forms of fibronectin carrying this sequence are not evenly distributed in the embryo, thus allowing migrating neural crest cells to orientate in the embryo. Fibronectin would then not only play a permissive role in embryonic cell motility, but have an instructive function in cell behavior.

morphogenesis / extracellular matrix / fibronectin / cell migration / neural crest

\section{INTRODUCTION}

Chez les vertébrés, mais aussi chez les invertébrés supérieurs, la genèse de la forme est probablement un des faits les plus marquants et les plus importants de la vie embryonnaire. En effet, la mise en place des tissus détermine la transmission des signaux nécessaires à la différenciation; de plus, la forme d'un organe conditionne très souvent sa fonction et la forme d'un organisme est adaptée à son mode de vie.

Comment s'effectuent ces transformations de l'ovocyte à l'organisme adulte ? Si l'on considère des organes aussi divers que le cerveau, le foie, le ccur ou les os, on s'aperçoit que les stratégies employées par l'embryon pour aboutir à leur formation diffèrent énormément d'un organe à l'autre et d'une espèce animale à l'autre. II n'est donc pas possible de donner une description, commune à tous les organismes, de l'ensemble des phénomènes de différenciation des tissus embryonnaires. On peut cependant rechercher des mécanismes de base qui se retrouvent dans la genèse de tous les tissus. On se rend compte ainsi qu'un même tissu peut subir, au cours de sa vie embryonnaire, de multiples remaniements successifs, allant d'une simple prolifération locale d'une partie de ses cellules à sa dislocation complète suivie de l'émigration de ses cellules dans des régions variées de l'individu. On a pu ainsi déterminer environ 6 processus primaires impliqués dans les phénomènes de morphogenèse et d'histogenèse. II s'agit, d'une part de la prolifération, de la mort et de la différenciation cellulaire. Ces processus participent de manière importante à la morphogenèse mais ils ne permettent pas généralement la mise en place des tissus. Celle-ci s'effectue surtout grâce aux processus de reploiements ou de dédoublements de feuillets simples en strates et en lobes (et, à l'échelle de l'individu, de reploiements d'un ensemble de tissus), de conversions épithélio-mésenchymateuses et de migrations d'ensembles de cellules ou de cellules isolées.

Ces différents processus morphogénétiques ne sont pas le fruit du hasard mais font partie d'une chaîne continue et ordonnée d'événements; ils concernent des territoires précis de l'embryon et interviennent 
à des moments donnés du développement. L'existence d'un remaniement tissulaire dépend donc très souvent du bon déroulement de ceux qui le précèdent.

Par quels mécanismes s'effectuent les conversions épithéliomésenchymateuses ? Comment certaines cellules parviennentelles à se déplacer dans l'embryon alors que d'autres restent immobiles dans le même environnement ? On peut approcher ce problème par la détermination des éléments cellulaires impliqués lors des remaniements tissulaires. Ces processus peuvent en effet engendrer des transformations parfois considérables dans l'organisation et la physiologie cellulaires. Ainsi, lors de la conversion d'un épithélium en mésenchyme, on assiste à des changements dans la forme et la polarité de la cellule, dans l'organisation de son cytosquelette et dans son mode d'interaction avec les cellules et la matrice extracellulaire présentes dans son voisinage. II importe donc de comprendre l'agencement des différents éléments impliqués dans les interactions entre les compartiments extracellulaires, membranaires et intracellulaires, et de suivre leur destinée au cours des remodelages tissulaires.

Parmi les nombreux composants dont dispose une cellule pour interagir avec son environnement, il existe une famille de molécules, les molécules adhésives, plus particulièrement impliquées dans l'adhérence directe de la cellule, soit avec ses voisines, soit avec la matrice extracellulaire. Certaines de ces molécules se retrouvent uniquement associées aux cellules épithéliales; d'autres sont communes aux cellules épithéliales et aux cellules mésenchymateuses, mais leur distribution à la surface cellulaire varie suivant le type de cellule rencontré. De telles différences laissent supposer que les molécules adhésives sont directement impliquées dans les processus de remaniements tissulaires.
Dans cette brève synthèse, nous nous limiterons à l'examen du rôle de certains éléments de la matrice extracellulaire dans un exemple de migration cellulaire au cours du développement : les crêtes neurales.

\section{LA FIBRONECTINE, UNE FAMILLE MAJEURE DE MOLÉCULES DES MATRICES EXTRACELLULAIRES IMPLIQUÉES DANS L'ADHÉRENCE}

Les molécules d'adhérence aux matrices extracellulaires sont en fait constituées par des couples de glycoprotéines : l'un des 2 éléments est intégré à la membrane plasmique de la cellule et sert de récepteur à l'autre composant du couple qui, lui, fait partie de la matrice extracellulaire. On a recensé, jusqu'à présent, de nombreuses molécules qui permettent l'adhérence des cellules aux matrices extracellulaires. $\mathrm{Ce}$ pendant, certaines d'entre elles sont restreintes à des tissus hautement différenciés; elles ne peuvent donc pas prétendre jouer un rôle majeur dans les processus de remodelage tissulaire chez le jeune embryon. Pour l'heure, seules les molécules ubiquitaires comme la fibronectine, la vitronectine, la laminine, la cytotactine et les collagènes, sont susceptibles d'être impliquées. Nous ne considérerons que l'une d'entre elles, la fibronectine, dont nous rappelerons les faits saillants.

De par sa quantité et ses fonctions, la fibronectine est un des éléments majeurs des espaces extracellulaires (pour synthèse, voir Mosher, 1988). Elle est constituée d'une famille de glycoprotéines présentes sous forme de fibres au sein des tissus conjonctifs et dans les membranes basales des épithéliums, et à l'état soluble dans les liquides plasmatique, cérébrospinal, synovial et amniotique. Les fonctions primaires de la fibronectine fibrillaire (ou cellulaire) sont d'organiser les matrices ex- 
tracellulaires et de promouvoir l'attachement, l'étalement et le déplacement cellulaires. La fibronectine du plasma, quant à elle, est impliquée dans la formation des caillots sanguins et dans l'activité de phagocytose des macrophages.

\section{Structure des molécules et du gène}

Toutes les molécules de fibronectine sont composées de 2 sous-unités très similaires, de masse moléculaire de $220 \mathrm{kDa}$ environ et reliées entre elles par 2 ponts disulfures. Elles sont codées par 1 seul gène de grande taille (70 kb), qui contient 48 exons (pour synthèse, voir Hynes, 1985). La différence entre les diverses formes de fibronectine réside avant tout dans la présence ou l'absence de plusieurs séquences au sein de la molécule; ces séquences sont chacune codées par un exon qui peut être épissé de manière différentielle au cours de la maturation des ARN messagers précurseurs (fig 1). Ainsi, seules les formes cellulaires de la fibro- nectine comportent les 2 séquences appelées EIIIA et EIIIB situées au milieu de chaque sous-unité (Kornblihtt et al, 1984, 1985; Paul et al, 1986). Une troisième séquence, la région IIICS présente du côté C-terminal des sous-unités, peut être épissée en plusieurs endroits, ce qui engendre au moins 5 molécules différentes. Cette séquence se retrouve tant sur les formes plasmatiques que cellulaires de la fibronectine.

Les recherches actuelles s'orientent surtout sur la signification biologique de ces séquences et des différentes formes de fibronectine. Malheureusement, jusqu'à présent, aucune fonction particulière ne semble associée aux régions EIIIA et EllIB. Cependant, des travaux récents utilisant des anticorps monoclonaux ont montré une forte incidence de ces 2 séquences dans la fibronectine synthétisée par des lignées tumorales. Ceci suggère que la transformation cellulaire pourrait induire des changements dans l'épissage des ARN messagers codant pour la fibronectine (Borsi et al, 1987). On peut donc ima-
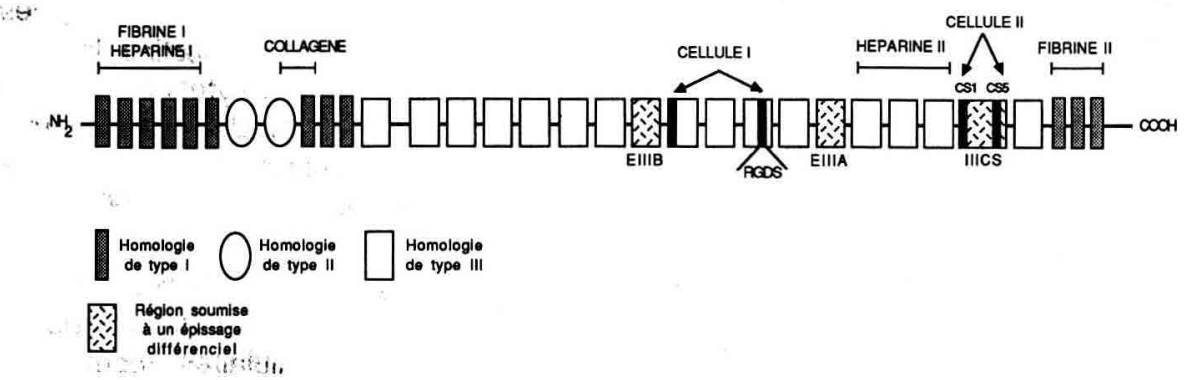

Fig 1. Représentation schématique d'une chaîne de fibronectine montrant la localisation des différents domaines de liaisons (indiqués par des rectangles noirs) pour les macromolécules des matrices extracellulaires et pour la surface cellulaire. II existe 2 principaux domaines de liaison à la cellule. Le premier est constitué de 2 sites séparés dont l'un contient la séquence RGDS (Arg-Gly-Asp-Ser) et dont l'autre, de séquence encore inconnue, est situé près du domaine EllIB. Ces 2 sites fonctionnent, de manière indépendante, mais aussi en synergie, afin de garantir une liaison complète avec la surface cellulaire. Le $2^{\theta}$ domaine de liaison à la cellule est lui aussi composé de 2 sites appelés CS1 et CS5 tous 2 situés dans la région lilCS. Ces 2 sites sont également indépendants mais peuvent fonctionner de façon additive. Le schéma illustre aussi les différentes homologies trouvées le long de la molécule, ainsi que celles qui sont soumises à des épissages différenciels. 
giner une régulation fine et hautement spécifique du comportement cellulaire par les diverses formes de fibronectine. L'établissement d'une cartographie précise des différentes formes dans les tissus, tant chez l'embryon que chez l'adulte, grâce à l'utilisation d'anticorps monclonaux ou de sondes génomiques spécifiques de chacune des formes, constitue un autre aspect important de ces recherches.

\section{Domaines fonctionnels de la fibronectine}

Les multiples fonctions de la fibronectine résultent de la présence, le long de la molécule, de domaines de liaison pour d'autres composants des espaces extracellulaires et pour la surface cellulaire (fig 1) (Hynes et Yamada, 1982). On trouve ainsi plusieurs domaines d'affinités diverses pour l'héparine et la fibrine et 1 pour le collagène. Ces domaines fonctionnels correspondent à des associations de domaines structuraux, appelés homologies de type I, II ou III; ils sont séparés par des régions très sensibles à l'action des protéases. Ainsi, par exemple, le site de liaison au collagène a été cartographié à la jonction d'une homologie de type II et d'une de type I, et la présence de ponts disulfures à l'intérieur de ces homologies est requise pour cette activité de liaison (Owens et Baralle, 1986).

L'interaction de la fibronectine avec la surface cellulaire s'effectue par le biais de plusieurs séquences situées dans la moitié C-terminale de chaque sous-unité. Le site majeur de liaison est porté par une homologie de type III et possède la séquence RGDS (arginine-glycine-aspartate-sérine). Cependant, jusqu'à très récemment, on ne parvenait pas à comprendre pourquoi le peptide RGDS, ou un fragment de 11,5 $\mathrm{kDa}$ le comprenant, a une affinité pour la surface cellulaire beaucoup plus faible que la fibronectine intacte (respectivement $K_{A}$ $=10^{4} \mathrm{M}^{-1}$ et $\mathrm{K}_{\mathrm{A}}=0,8 \times 10^{6} \mathrm{M}^{-1}$; Akiyama et al, 1985). Par des techniques de biologie moléculaire, il a été possible de montrer qu'une région de la molécule, située loin en amont du site RGDS, est nécessaire pour obtenir une affinité équivalente à celle de la protéine d'origine (Obara et al, 1988). Le domaine de liaison à la cellule se compose donc de 2 séquences séparées l'une de l'autre et fonctionnant en synergie.

Les autres domaines de liaison pour la cellule sont portés par la région IIICS et peuvent être utilisés par certains types cellulaires tels que les mélanomes (Humphries et al, 1986, 1987). L'un, de séquence REDV (arginine-glutamateaspartate-valine) et de faible affinité, y est situé à l'extrémité C-terminale. L'autre, de séquence non encore précisée et de haute affinité, s'y trouve dans l'extrémité $\mathrm{N}$ terminale. Ces 2 domaines de liaison peuvent agir indépendamment ou en synergie (Humphries et al, 1987).

\section{Les récepteurs de la fibronectine}

Le principal récepteur de la fibronectine purifié à ce jour est un complexe de glycoprotéines membranaires de $140 \mathrm{kDa}$ de masse moléculaire, qui appartient à la famille des intégrines (pour synthèses, voir Hynes, 1987; Buck et Horwitz, 1987). Cette famille des intégrines est constituée de glycoprotéines membranaires liant diverses molécules des espaces extracellulaires et construites sur un modele commun de 2 chaînes appelées $\alpha$ et $\beta$ (fig 2).

La séquence et l'organisation dans la membrane des chaînes $\alpha$ et $\beta$ sont maintenant connues (Tamkun et al, 1986; Argraves et al, 1986; Suzuki et al, 1986). Ce sont des protéines transmembranaires as- 


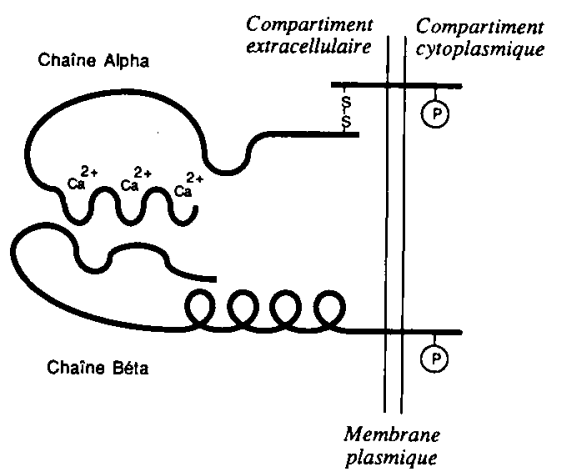

Fig 2. Représentation schématique du récepteur des fibronectines. Le récepteur des fibronectines, qui appartient a la famille des intégrines, est constitué d'une chaîne $\alpha$ et d'une chaîne $\beta$ associées de façon non covalente dans leur partie $\mathrm{N}$-terminale. Elles possèdent toutes 2 une courte séquence cytoplasmique. La chaîne $\alpha$, de masse moléculaire $160 \mathrm{kDa}$ environ, est composée d'un court fragment transmembranaire relié au fragment extracellulaire par un pont disulfure. Cette chaine contient, en outre, dans sa partie $\mathrm{N}$-terminale, des domaines de liaison aux ions calcium. La chaîne $\beta$, de $120 \mathrm{kDa}$, est caractérisée par la présence de séquences riches en acide aminé cystéine imprimant à ces régions la forme de boucles. Les 2 chaînes possedent enfin dans leur partie Cterminale des sites de phosphorylation potentiels.

sociées l'une à l'autre dans leur partie la plus extracellulaire. Les sous-unités $\beta$ sont au nombre de 3 , très similaires, d'un récepteur à l'autre et d'une espèce à l'autre. Elles possèdent un domaine riche en cystéines, qui leur imprime une structure hélicoidale. Les sous-unités $\alpha$ sont plus sujettes à des variations que les sous-unités $\beta$, et on pense qu'elles assurent la spécificité de liaison des récepteurs. Cependant, elles possèdent toutes des caractéristiques communes, en particulier un court fragment transmembranaire relié au reste de la molécule par un pont disulfure, ainsi que des domaines de liaison au calcium dans la région $\mathrm{N}$-terminale. Le domaine de liaison du récepteur à son ligand n'est pas précisément localisé sur les sous-unités mais la présence des 2 chaînes est nécessaire à la fonction de liaison (Buck et al, 1986). De plus, certains de ces récepteurs (comme le récepteur de la fibronectine et celui de la vitronectine) reconnaissent la séquence RGD sur leur ligand.

Les récepteurs de la fibronectine constituent un maillon important dans la chaîne qui relie le compartiment cytoplasmique d'une cellule au milieu extracellulaire. En effet, il est principalement concentré dans les points d'ancrage de la cellule à son support (Chen et al, 1985a; Damsky et al, 1985) où il interagit avec la taline, un composant du cytosquelette (Horwitz et al, 1986). Une telle position à l'interface des 2 compartiments prédestine les récepteurs de la fibronectine à un rôle clé dans le contrôle du comportement cellulaire.

L'interaction entre les molécules de la fibronectine et leurs récepteurs peut être modulée de diverses manières. Tout d'abord, dans les cellules transformées, la région intracytoplasmique des chaînes $\alpha$ et $\beta$ peut être phosphorylée, ce qui provoquerait une rupture de la liaison entre le récepteur et ses ligands (Hirst et al, 1986). D'autres composants des espaces extracellulaires, comme les collagènes ou l'héparine, peuvent plus ou moins directement modifier l'affinité du récepteur pour son ligand (Laterra et al, 1983; Nagata et al, 1985). Enfin, il est apparu récemment que l'expression des récepteurs de la fibronectine peut être contrôlée par des facteurs de croissance tels que le TGB $\beta$ (lgnotz et Massagué, 1987).

\section{LES CRÉTES NEURALES, UN MODĖLE DE MORPHOGENESE EMBRYONNAIRE}

Les crêtes neurales sont une population cellulaire qui se développe à partir du neu- 
rectoderme à l'issue de la neurulation. De cette population apparemment homogène, dérive un nombre très important de types cellulaires : la plupart des neurones et toutes les cellules gliales du système nerveux périphérique, la totalité des cellules pigmentaires a l'exception de celles de la rétine, des cellules neuroendocrines comme celles de la médullosurrénale, du corps carotidien et du corps ultimobranchial et enfin, dans la tête et le cou, une très grande partie de cellules osseuses, musculaires et conjonctives (appelées dérivés mésectodermiques) ainsi que les méninges (pour synthèse et références, voir Le Douarin, 1982). La grande particularité des crêtes neurales, outre la variété des cellules qui en dérivent, tient au fait que ses cellules émergent d'une région restreinte du neurectoderme (l'interface entre l'ectoderme et le neuro-épithélium, le long de l'axe de l'embryon) et quittent leur site d'origine pour entamer une migration divergente, souvent fort longue, dans diverses régions de l'embryon. Dans le tronc, cette migration s'achève surtout par la formation des rudiments des ganglions périphériques et, dans la tête, les cellules des crêtes neurales investissent les parties ventrales de l'embryon où elles s'accumulent pour donner les tissus mésectodermiques (pour synthèses, voir Weston, 1982; Le Douarin, 1982).

On peut donc ainsi distinguer 3 étapes dans le développement des crêtes neurales avant leur différenciation finale. La première est la phase d'émigration pendant laquelle les cellules se dissocient de l'épithélium neural et entament leur migration. La deuxième étape est la migration proprement dite. L'arrêt des cellules et leur réassociation avec leurs voisines pour constituer les ébauches des tissus qui en dérivent, forment la troisième étape. Ces étapes peuvent donc se résumer en une succession de conversions épithélio- mésenchymateuses associées à des migrations cellulaires. Nous nous limiterons à l'étude du rôle des fibronectines au cours de la seule phase de migration.

Quand on transplante des cellules fibroblastiques dans les voies de migration des cellules des crêtes neurales, on observe qu'elles restent immobilisées dans la région où elles ont été greffées; inversement, si l'on répète la même expérience avec des cellules des crêtes neurales issues d'autres régions de l'embryon ou des mélanocytes ou encore des lignées cellulaires transformées, on s'aperçoit qu'elles migrent et se distribuent dans les mêmes sites que le feraient les cellules des crêtes neurales de l'hôte (Erickson et al, 1980; Erickson, 1985). On peut donc en déduire que :

- certaines cellules possèdent la capacité intrinsèque de se mouvoir;

- l'environnement ne peut induire un comportement de migration à lui seul, mais permet aux cellules de rejoindre leur territoire cible. II est donc nécessaire de déterminer les composantes de l'environnement qui guident les cellules et aussi le mode d'interaction de la cellule en mouvement avec ces composantes.

\section{Voies de migration : structure et configuration}

Au sortir du tube nerveux, les cellules des crêtes neurales entrent en relation avec un environnement dont l'architecture varie à chaque niveau du névraxe; de plus, dans la majorité des cas, les aires où les cellules s'accumulent à la fin de leur migration ne sont aisément identifiables ou n'existent pas au moment où elles entament leur migration. II s'avère donc nécessaire de suivre pas à pas le déplacement des cellules, au cours du temps et à chaque niveau de l'embryon. 
La durée de la migration des cellules des crêtes neurales pouvant être parfois considérable (plusieurs jours le long du tube digestif, chez l'oiseau), des marqueurs spécifiques sont indispensables pour suivre ces cellules. Divers marqueurs dont l'utilisation repose sur 3 principes différents ont été utilisés. Le premier cas consiste à transplanter des tubes nerveux ou des fragments de tube nerveux marqués de telle manière que les cellules greffées puissent être repérées aisément : marquage préalable des tissus par la thymidine tritiée (Weston, 1963; Noden, 1975) ou construction de chimères interspécifiques entre la caille et la poule (pour synthèse, voir Le Douarin et McLaren, 1984). Ces techniques se sont révélées très efficaces pour suivre les cellules et surtout pour établir la liste de leurs dérivés; cependant, le premier type de marquage a l'inconvénient de n'être que temporaire, car il se dilue rapidement par suite des divisions cellulaires successives.

Dans le deuxième cas, il s'agit de rechercher des marqueurs intrinsèques aux cellules en déplacement. Le premier exemple est l'acétylcholinestérase (Cochard et Coltey, 1983). Cependant, la spécificité de ce marqueur n'est pas totale, surtout quand les cellules arrivent au terme de leur migration. Le deuxième exemple est l'utilisation d'anticorps monoclonaux spécifiques. Nous avons isolé un anticorps monoclonal, NC-1, identique à l'anticorps HNK-1 et qui reconnaît les cellules des crêtes neurales en migration. Cet anticorps marque les cellules peu après leur sortie du tube nerveux. L'épitope reconnu reste exprimé sur la quasi-totalité des cellules au cours de leur migration, mais aussi sur la grande majorité des neurones et cellules du soutien du système nerveux périphérique. A l'opposé, les cellules qui donnent les dérivés mésectodermiques le perdent rapidement, une fois arrivées à leur site d'arrêt (Vincent et Thiery, 1984).

La troisième approche pour suivre les cellules des crêtes neurales dans leur migration consiste à établir la structure et l'organisation des routes qu'elles empruntent à l'aide de divers marqueurs (voir ciaprès). Quelle que soit la méthode de traçage utilisée, avec ses avantages et ses limites, elle fournit des résultats identiques à ceux des autres (Le Douarin et al, 1984).

Les voies de migration des cellules des crêtes neurales varient en fonction du niveau axial considéré et en fonction du stade de développement de l'embryon (Tosney, 1978, 1982; Duband et Thiery, 1982; Thiery et al, 1982). Cependant, on observe une grande constance dans leur configuration, du fait même de l'organisation de l'embryon. En effet, comme ce dernier est surtout constitué d'épithéliums se faisant face, de nombreux espaces acellulaires restreints et bien délimités sont ménagés, qui forment des couloirs. Au cours de leur migration, les cellules des crêtes neurales empruntent ces espaces limités qui leur permettent de rejoindre leurs territoires cibles. La morphologie de ces voies évolue au cours de la migration des cellules des crêtes neurales et, dans certains cas, la disparition des couloirs de migration correspond à l'arrêt des cellules.

D'une manière générale, les cellules des crêtes neurales migrent suivant les directions dorso-ventrale et-ou dorsolatérale et elles ne s'étendent que peu suivant l'axe antéro-postérieur (voir aussi Teillet et al, 1987). Cependant, ce patron peut être localement modifié dans la tête et le cou par la présence d'obstacles qui obligent les cellules à se répartir dans des régions antérieures et-ou postérieures à leur site d'origine. C'est le cas, par exemple, des vésicules optiques, des placodes optiques ou encore des placodes pharyngiennes (fig 3). 
Le rôle de guide imposé par l'environnement est particulièrement évident dans la région troncale où la métamérie du mésoderme somitique se reflète dans la répartition des dérivés des crêtes neurales (fig 4). II existe 2 axes principaux de migration, l'un entre les somites consécutifs et l'autre, le long des somites, contre le tube nerveux. Ces 2 axes seraient en grande partie responsables de la séparation des lignages sympathiques et sensoriels. Plus récemment, l'emploi des anticorps HNK-1 ou NC-1, ainsi que des chimères interspécifiques, a permis d'affiner cette cartographie des voies de migration des crêtes neurales dans la région troncale (Rickmann et al, 1985; Bronner-Fraser, 1986; Teillet et al, 1987). Les cellules qui migrent au niveau d'un somite ne se répartissent pas de manière uniforme par rapport à ce dernier : d'une part, certaines d'entre elles s'accumulent dans la partie rostrale du somite, entre le sclérotome et le tube nerveux, pour y donner les ganglions spinaux; d'autre part, toujours dans la partie rostrale du somite, une autre sous-population des crêtes neurales contourne le sclérotome le long de la face ventrale du myotome pour se loger dans la région aortique. Certaines de ces cellules migreront ensuite le long de l'aorte et se mêleront aux autres cellules qui auront migré dans l'espace intersomitique pour fournir les ganglions sympathiques et les plexus aortiques. D'autres s'associeront aux nerfs moteurs en migration pour devenir des cellules de Schwann.

Les raisons qui poussent les cellules des crêtes neurales à n'occuper que la partie rostrale du somite ne sont pas encore élucidées, d'autant plus que leur déplacement est imbriqué avec l'expansion du sclérotome autour du tube nerveux. La plupart des composants des matrices extracellulaires $y$ ont en effet la même répartition que dans la partie caudale, à part la cytotactine qui est surtout présente du côté caudal (Tan et al, 1987) et qui pourrait
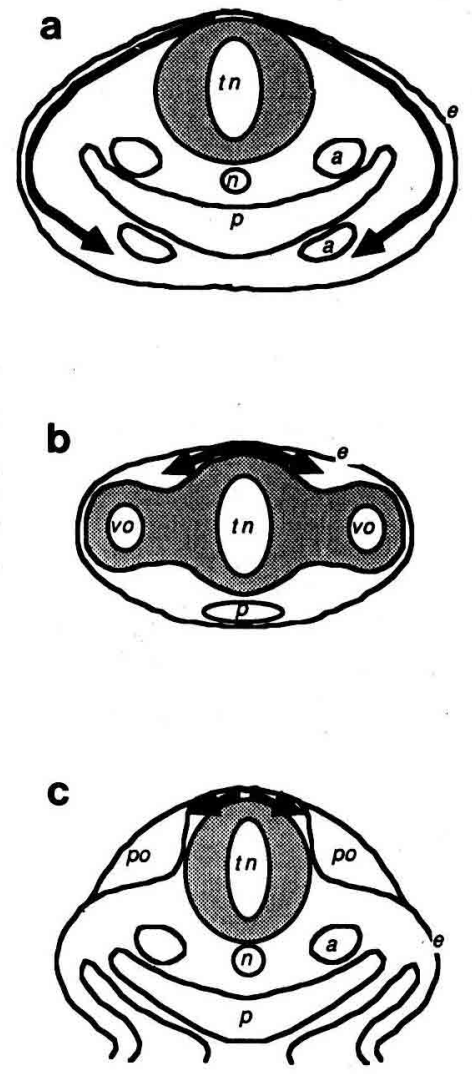

Fig 3. Principales voies de migration des cellules des crêtes neurales dans la tête. a mésencéphale. Les cellules des crêtes neurales empruntent une voie latéro-ventrale sous l'ectoderme pour rejoindre les arcs branchiaux où elles se différencieront principalement en tissu conjonctif, muscles et cartilage. $b$ et $\mathbf{c}$ régions des vésicules optiques et des placodes optiques. Dans ces régions, la voie latéro-ventrale typique est obstruée par l'excroissance du tube nerveux pour donner les vésicules optiques ou par l'épaississement de l'ectoderme constituant les placodes optiques. II en résulte que les cellules des crêtes neurales sont momentanément bloquées. Leur migration ne reprendra qu'avec le contournement de ces obstacles. $a$ : aorte; $\theta$ : ectoderme; $n$ : notochorde; $p$ : pharynx; $p o$ : placode optique; tn : tube nerveux; vo : vésicule optique. Les flèches indiquent les voies de migration des crêtes neurales. 
(a)

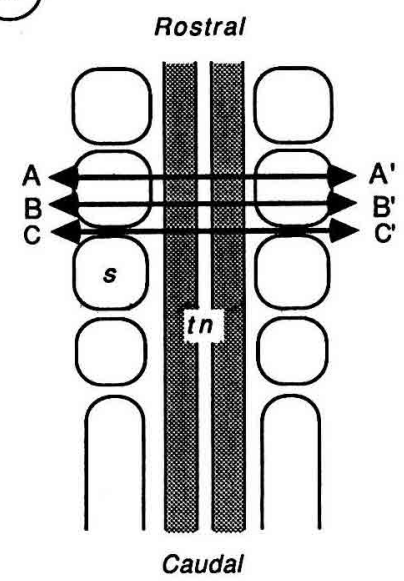

b)

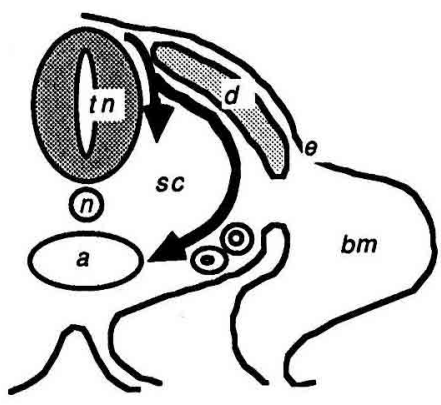

Coupe A-A'

(C)

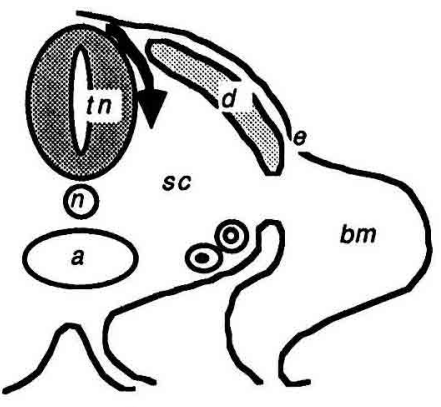

Coupe B-B'

(d)

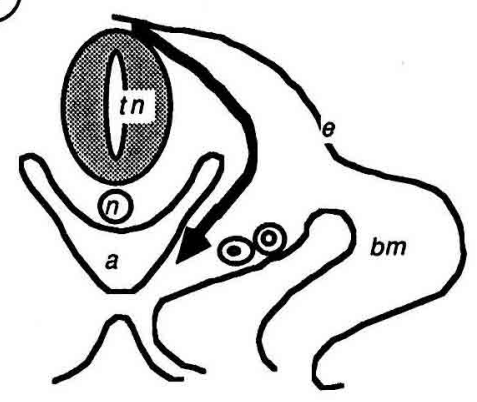

Coupe C-C'

Fig 4. Principales voies de migration des cellules des crêtes neurales dans le tronc. En a sont représentés les niveaux des coupes effectuées au travers d'un somite et illustrées en b, $\mathbf{c}$ et $\mathbf{d}$. b se situe dans la partie rostrale du somite. Les cellules des crêtes neurales migrent ventralement le long du tube nerveux et du dermomyotome pour former, d'une part le ganglion sensoriel et les cellules de Schwann associées au nerf moteur, et d'autre part les chaînes sympathiques. c se situe dans la moitié caudale du somite. Dans cette région, les cellules restent bloquées le long de la partie dorsale du tube nerveux. d se situe entre 2 somites consécutifs. Les cellules des crêtes neurales migrent directement dans l'espace intersomitique vers la région périaortique où elles donneront les chaînes sympathiques. a : aorte; $b m$ : bourgeon de membre; $d$ : dermomyotome; $\theta$ : ectoderme; $n$ : notochorde; $s$ : somite; sc : sclérotome; $t n$ : tube nerveux. 
donc canaliser les cellules des crêtes neurales vers la région rostrale des somites.

Les espaces acellulaires présents aux stades de la migration des cellules des crêtes neurales contiennent de la fibronectine, du collagène de type I, de la cytotactine, et sont limités par la laminine et les collagènes des lames basales des épithéliums. C'est dans cet ensemble de glycoprotéines que migrent les cellules des crêtes neurales (Duband et Thiery, 1982, 1987; Thiery et al, 1982; Tan et al, 1987). En outre, ces voies de migration contiennent de l'acide hyaluronique et des protéoglycannes. Cependant, si l'on examine la répartition des cellules des crêtes neurales, on s'aperçoit qu'elles sont toujours associées à de la fibronectine et à du collagène de type I, mais qu'elles n'occupent pas tous les espaces possibles. II existe donc des restrictions à leur passage. Les causes de telles restrictions ne sont pas toutes connues mais elles peuvent, dans certains cas, dépendre de la présence de cytotactine et du taux de chondroïtine sulfate.

\section{Comportement migratoire des cellules des crêtes neurales}

\section{Interaction avec les constituants des matrices}

Avec quels composants des matrices extracellulaires les cellules des crêtes neurales interagissent-elles pour se déplacer? Une telle détermination a été obtenue en culture in vitro et par des expériences de perturbation. Ces cellules sont capables d'adhérer à des substrats plans de fibronectine, de s'y étaler et d'y migrer (Newgreen et al, 1982; Rovasio et al, 1983). La laminine présente une capacité plus réduite à promouvoir le déplacement des crêtes neurales, surtout à leur départ du tube nerveux (Rovasio et al, 1983). Le collagène de type I, l'acide hyaluronique, divers protéoglycanes sulfatés et la cytotactine ne permettent ni leur étalement ni leur migration (Newgreen et al, 1982; Rovasio et al, 1983; Tan et al, 1987). Si l'expérience est reproduite sur des substrats tridimensionnels (Tucker et Erickson, 1984), on obtient essentiellement les mêmes résultats, sauf que le collagène de type I peut favoriser un déplacement appréciable des cellules, mais toujours dans une moindre mesure que la fibronectine, et à faible concentration seulement. L'association collagène-fibronectine se révèle être un substrat particulièrement efficace, ce qui est à rapprocher de la situation observée in vivo.

Diverses expériences de perturbation, par inhibition stérique ou par inhibition compétitive, ont prouvé que l'interaction directe entre la fibronectine et la surface des cellules des crêtes neurales est nécessaire pour leur migration. Des anticorps, dirigés contre un fragment de fibronectine qui contient le domaine de liaison à la cellule, inhibent de manière réversible le comportement migratoire des cellules, tant in vivo qu'in vitro (Rovasio et al, 1983; Poole et Thiery, 1987). De même, pour des anticorps dirigés contre la chaîne $\beta$ des récepteurs de la fibronectine (Bronner-Fraser, 1985; Duband et al, 1986). Enfin, des décapeptides qui incluent la séquence RGDS du site de liaison à la cellule possèdent le même effet inhibiteur (Boucaut et al, 1984).

\section{Organisation de la machinerie de locomotion}

Nous avons recherché la cause de la différence du comportement des cellules en mouvement et de celles en état stationnaire, dans l'organisation du cytosquelette et des points d'ancrage au substrat (fig 5). 
En effet, ces structures doivent, dans un cas, permettre à la cellule une interaction provisoire avec son substrat pour établir de nouveaux contacts et, dans l'autre cas, elles doivent au contraire garantir à la cellule un ancrage fort à son substrat.

Quand elles se déplacent, les cellules des crêtes neurales ne synthétisent et ne déposent pas de fibronectine sur leur substratum (Newgreen et Thiery, 1980; Ffrench-Constant et Hynes, 1988). Le récepteur de la fibronectine est réparti de manière diffuse sur toute la surface membranaire de ces cellules et les points d'ancrage au substrat ont une organisation particulière (Duband et al, 1986). En effet, on observe peu de points de contact focaux, l'adhérence au substratum étant uniforme. La taline, la vinculine et l' $\alpha$-actine sont surtout à l'état diffus dans le cytoplasme (Duband et al, 1986). Enfin, les microfilaments d'actine ne forment pas de câbles organisés et se concentrent dans la périphérie cellulaire, suivant le grand axe de la cellule. A l'inverse, les cellules stationnaires déposent autour d'elles un réseau dense de fibres de fibronectine. Elles adhèrent à leur substratum en de nombreux points de contact focaux où se concentrent le récepteur de la fibronectine, la vinculine, la taline et l' $\alpha$-actinine. L'actine forme des réseaux de câbles qui irradient à partir de la région nucléaire, jusque dans la périphérie de la cellule (Duband et al, 1986).

Dans les cellules immobiles, la concentration du récepteur de la fibronectine dans des points précis de la cellule, où viennent s'attacher les fibres du cytosquelette et les constituants de la matrice extracellulaire, augmenterait la force d'attachement de la cellule au substratum et compenserait ainsi la faiblesse de l'interaction entre le récepteur et son ligand. Au contraire, dans les cellules en mouvement, la relative dé-sorganisation du cytosquelette assurerait la plasticité de l'architecture de la cellule et la répartition du récepteur sur l'ensemble de la surface cellulaire permettrait un attachement transitoire de la cellule, à son substratum. De plus, la distribution du récepteur dans les cellules mobiles et stationnaires suggère que, dans le deuxième cas, il est lié fortement au cytosquelette (ce qui le rend immobile dans le plan de la membrane) tandis que, dans le premier cas, il ne s'y lie que de façon transitoire (et il serait donc mobile). L'utilisation de la technique de récupération de la fluorescence après photoextinction nous a permis de confirmer cette hypothèse (Duband et al, 1988a). Dans les cellules mobiles, une grande majorité des récepteurs de la fibronectine peuvent se déplacer dans le plan de la membrane, alors que, dans les cellules immobiles, ils sont immobiles.

Pour se déplacer, les cellules ne doivent pas adhérer trop fortement à leur substratum. La forte proportion de récepteurs mobiles est en une première indication. Une autre indication est fournie par l'étude du comportement des cellules sur des feuilles de silicones. En effet, les propriétés des feuilles de silicones permettent d'estimer d'une manière simple la force

Fig 5. Modèles spéculatifs comparant l'organisation des points d'ancrage au substrat de cellules immobiles a et migratrices b. Alors que dans les cellules immobiles, les éléments participant à l'adhérence cellulaire sont concentrés dans certaines régions de la cellule de manière à pourvoir un ancrage fort au substrat, dans les cellules en locomotion, ces mêmes éléments sont en constante mobilité afin de permettre à la cellule de se déformer rapidement et de se détacher du substrat. En particulier, les récepteurs des fibronectines sont, à la fois mobiles dans le plan de la membrane, et peuvent être activement internalisés pour être, soit dégradés (voie 1), soit transportés à travers le cytoplasme, recyclés dans la membrane et enfin être réutilisés pour former de nouveaux points d'ancrage au substrat (voie 2). 
(A) Etat immobile

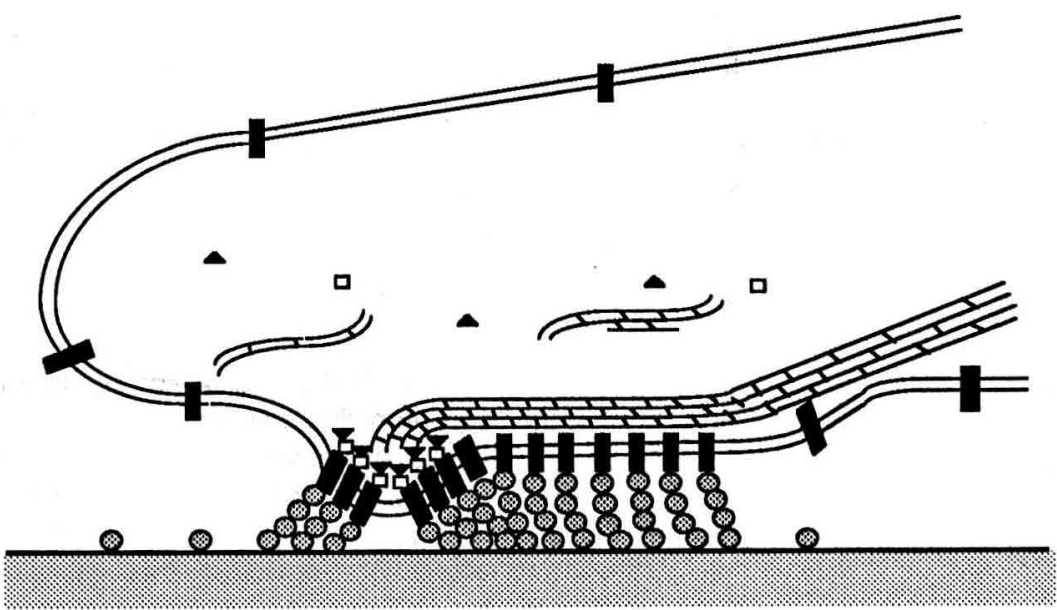

B) Etat migratoire

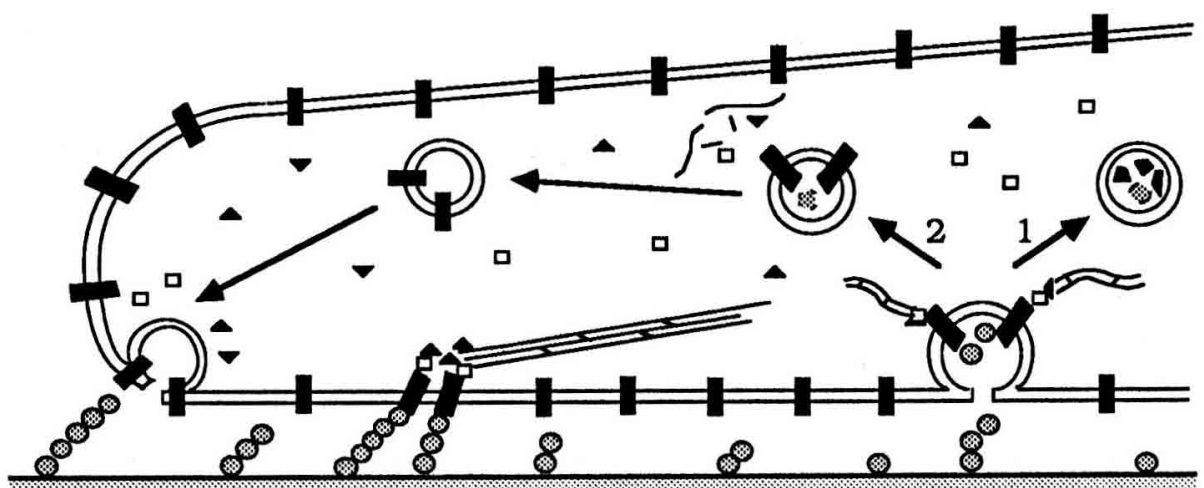
- Fibronectine
Récepteur
fibronectine
$\square$ Taline $\nabla$ Vinculine
alpha-actinine
alpha-actinine
icrofilaments d'actine 
d'adhérence d'une cellule sur son substratum. Ainsi, à l'inverse des cellules stationnaires, les cellules des crêtes neurales n'impriment aucune déformation à ces feuilles (Tucker et al, 1985). Pour notre part, nous avons mesuré le déplacement cellulaire sur un substratum d'anticorps dirigés contre le récepteur de la fibronectine; l'affinité des anticorps pour leur ligand est environ 100 fois supérieure à celle des fibronectines pour leurs récepteurs. Si les cellules peuvent adhérer parfaitement sur un tel substratum, elles s'y déplacent très lentement et forment des plages pseudoépithéliales (Duband et al, 1986).

Quel est le mécanisme qui intervient pour réguler l'interaction transitoire entre le récepteur de la fibronectine et son ligand? A cet égard, il est intéressant de noter que l'ensemble des propriétés des cellules embryonnaires douées de motilité est à rapprocher de celles des cellules transformées (Yamada et al, 1976; Chen et al, 1986). Or, chez ces cellules, le récepteur est phosphorylé, ce qui aurait pour effet de le dissocier de la taline et de la fibronectine (Hirst et al, 1986). Cependant, il n'a pas été possible de mettre en évidence une quelconque phosphorylation du récepteur dans les cellules des crêtes neurales, ce qui indique que la régulation de l'interaction récepteur-fibronectine passe par un autre procédé (Duband et al, 1988b). II est fort possible que cette régulation s'effectue par le biais d'une internalisation des récepteurs ancrés à la fibronectine, suivie de leur recyclage dans le cytoplasme (Duband et al, 1988c; Bretcher, 1989). Par ailleurs, les cellules transformées dégradent partiellement la matrice extracellulaire sur laquelle elles reposent (Chen et al, 1984; 1985b). Pour cela, elles utilisent des protéases associées à la membrane et qui se concentrent dans les sites d'ancrage de la cellule (Chen et al, 1984; Chen et Chen, 1987). Les cellules des crêtes neurales possèdent un comportement très proche; elles synthétisent de l'activateur du plasminogène (Valinsky et Le Douarin, 1985) et réorganisent la matrice au cours de leur déplacement (Rovasio et al, 1983). Une dégradation ménagée de la matrice leur permettrait d'accélérer à la fois leur détachement local du substrat et leur pénétration dans des réseaux de. fibres denses.

\section{CONCLUSION}

L'étude du rôle de la fibronectine dans la migration des cellules des crêtes neurales permet d'approcher la multiplicité des fonctions de cette protéine. Cependant, un certain nombre de questions restent à résoudre. En particulier, il serait intéressant de déterminer si la fibronectine constitue un substrat de migration commun à toutes les cellules embryonnaires en migration. L'examen des cellules germinales primordiales, du mésoderme durant la gastrulation, de l'endothélium cardiaque et des myoblastes suggère que ces cellules utilisent aussi la fibronectine comme substrat de migration, mais on ne sait pas pour l'heure si le mode d'interaction de ces cellules avec la fibronectine est similaire à celui décrit précédemment pour les crêtes neurales. Un autre aspect de ce problème est la multiplicité des récepteurs intégrines et il est fort possible qu'ils interviennent pour moduler l'activité de la fibronectine. Enfin, on peut se demander si ces récepteurs ne transduisent par des signaux au génome via des protéines $\mathrm{G}$ ou d'autres seconds messagers, affectant ainsi directement l'expression de certains gènes.

\section{RÉFÉRENCES}

Akiyama SK, Hasegawa E, Hasegawa T, Yamada KM (1985) The interaction of fibronectin 
fragments with fibroblastic cells. $J$ Biol Chem 260, 13256-13260

Argraves WC, Pytela R, Suzuki S, Millán JL, Pierschbacher MD, Ruoslahti E (1986) cDNA sequences from the alpha subunit of the fibronectin receptor predict a transmembrane domain and a short cytoplasmic peptide. $J$ Biol Chem 261, 12922-12924

Borsi L, Carnemolla B, Castellani P, Rosellini C, Vecchio D, Allemanni G, Chang SE, TaylorPapadimitriou J, Pande H, Zardi L (1987) Monoclonal antibodies in the analysis of fibronectin isoforms generated by alternative splicing of mRNA precursors in normal and transformed human cells. J Cell Biol 104, 595-600

Boucaut JC, Darribère T, Poole TJ, Aoyama H, Yamada KM, Thiery JP (1984) Biological active synthetic peptides as probes of embryonic development: a competitive peptide inhibitor of fibronectin function inhibits gastrulation in amphibian embryos and neural crest cell migration in avian embryos. $J$ Cell Biol 99, 1822-1830

Bretcher MS (1989) Endocytosis and recycling of the fibronectin receptor in $\mathrm{CHO}$ cells. $E M B O \mathrm{~J} 8,1341-1348$

Bronner-Fraser $M$ (1985) Alteration in neural crest migration by a monoclonal antibody that affects cell adhesion. J Cell Biol 101, 610617

Bronner-Fraser M (1986) Analysis of the early stages of trunk neural crest migration in avian embryos using monoclonal antibody HNK-1. Dev Biol 115, 44-55

Buck CA, Horwitz AF (1987) Cell surface receptors for extracellular matrix molecules. Annu Rev Cell Biol 3, 179-205

Buck CA, Shea E, Duggan K, Horwitz AF (1986) Integrin (the CSAT antigen): Functionality requires oligomeric integrity. $J \mathrm{Cell} \mathrm{Biol}_{103}$, 2421-2428

Chen JM, Chen WT (1987) Fibronectindegrading proteases from the membranes of transformed cells. Cell 48, 193-203

Chen WT, Olden K, Bernard BA, Chu FF (1984) Expression of transformation-associated protease(s) that degrade fibronectin at cell contact sites. J cell Biol 98, 1546-1555

Chen WT, Hasegawa E, Hasegawa T, Weinstock C, Yamada KM (1985a) Development of cell surface linkage complexes in cultured fibroblasts. J Cell Biol 100, 1103-1114

Chen WT, Chen JM, Parsons SJ, Parsons JT (1985b) Local degradation of fibronectin at sites of expression of the transforming gene product pp60src. Nature 316, 156-158

Chen WT, Wang J, Hasegawa T, Yamada SS, Yamada KM (1986) Regulation of fibronectin receptor distribution by transformation, exogenous fibronectin, and synthetic peptides. $J$ Cell Biol 103, 1649-1661

Cochard P, Coltey P (1983) Cholinergic traits in the neural crest: acetylcholinesterase in crest cells of the chick embryo. Dev Biol 98, 221228

Damsky CH, Knudsen KA, Bradley D, Buck CA, Horwitz AF (1985) Distribution of the cell substratum attachment (CSAT) antigen on myogenic and fibroblastic cells in culture. $J$ cell Biol 100, 1528-1539

Duband JL, Thiery JP (1982) Distribution of fibronectin in the early phase of avian cephalic neural crest cell migration. Dev Biol 93, 308323

Duband JL, Thiery JP (1987) Distribution of laminin and collagens during avian neural crest development. Development 101, 461-478

Duband JL, Rocher S, Chen WT, Yamada KM, Thiery JP (1986) Cell adhesion and migration in the early vertebrate embryo: location and possible role of the purative fibronectinreceptor complex. J Cell Biol 102, 160-178

Duband JL, Nuckolls GH, Ishihara A, Hasegawa $\mathrm{T}$, Yamada KM, Thiery JP, Jacobson $\mathrm{K}$ (1988a) Fibronectin receptor exhibits high lateral mobility in embryonic locomoting cells but is immobile in focal contacts and fibrillar streaks in stationary cells. J Cell Biol 107, 1385-1396

Duband JL, Dufour S, Yamada KM, Thiery JP (1988b) Phosphorylation of the fibronectinreceptor complex occurs during the acquisition of the stationary state by avian embryonic locomoting cells. FEBS Lett 230, 181-185

Duband JL, Dufour S, Thiery JP (1988c) Extracellular-matrix-cytoskeleton interactions in locomoting embryonic cells. Protoplasma 145, $112-119$

Erickson CA (1985) Control of neural crest cell dispersion in the trunk of the avian embryo. Dev Biol 111, 138-157 
Erickson CA, Tosney KW, Weston JA (1980) Analysis of migratory behavior of neural crest and fibroblastic cells in embryonic tissues. Dev Biol 77, 142-156

french-Constant C. Hynes RO (1988) Patterns of fibronectin gene expression and splicing during cell migration in chicken embryos. $\mathrm{De}$ velopment 104, 369-382

Hirst R, Horwitz A, Buck C, Rohschneider L (1986) Phosphorylation of the fibronectin receptor complex in cells transformed by oncogenes that encode tyrosine kinases. Proc Natl Acad Sci USA 83, 6470-6474

Horwitz A, Duggan K, Buck C, Beckerle MC, Burridge K (1986) Interaction of plasma membrane fibronectin receptor with talin. A transmembrane linkage. Nature 320, 531532

Humphries MJ, Akimaya SK, Komoriya A, Olden $K$, Yamada KM (1986) Identification of an alternatively-spliced site in human plasma fibronectin that possesses cell-type specificity. J Cell Biol 103, 2637-2647

Humphries M, Komoriya A, Akiyama SK, Olden K, Yamada KM (1987) Identification of two distinct regions of the type III connecting segment of human plasma fibronectin that promote cell type-specific adhesion. J Biol Chem 262, 6886-6892

Hynes RO (1985) Molecular biology of fibronectin. Annu Rev Cell Biol 1, 67-90

Hynes RO (1987) Integrins: A family of cell surface receptors. Cell 48, 549-554

Hynes RO, Yamada KM (1982) Fibronectins: Multifunctional modular glycoproteins. J Cell Biol 95, 369-377

Ignotz RA, Massagué J (1987) Cell adhesion protein receptors as targets for transforming growth factors- $\beta$ action. Cell 51, 189-197

Kornblihtt AR, Vibe-Pedersen $K$, Baralle FE (1984) Human fibronectin: Molecular cloning evidence for two mRNA species differing by an internal segment coding for a structural domain. EMBO J 3, 221-226

Kornblihtt AR, Umezawa K, Vibe-Pedersen K, Baralle FE (1985) Primary structure of human fibronectin: Differential splicing may generate at least 10 polypeptides from a single gene. EMBO J 4, 1755-1759

Laterra J, Silbert JE, Culp LA (1983) Cell surface heparan sulfate mediates some adhe- sive responses to glycosaminoglycan-binding matrices, including fibronectin. J Cell Biol 96, 112-113

Le Douarin NM (1982) The neural crest. Cambridge Univ Press, Cambridge

Le Douarin NM, McLaren A (1984) Chimeras in Developmental Biology. Acad Press, New York

Le Douarin NM, Cochard P, Vincent M, Duband JL, Tucker GC, Teillet MA, Thiery JP (1984) Nuclear, cytoplasmic, and membrane markers, to follow neural crest cell migration: $A$ comparative study. In: The role of extracellular matrix in development (Trelstad EL, ed) AR Liss, New York, 373-398

Mosher DF ed (1988) Fibronectin, Academic Press, New York

Nagata K, Humphries MJ, Olden K, Yamada KM (1985) Collagen can modulate cell interactions with fibronectin. J Cell Biol 101, 386394

Newgreen DF, Thiery JP (1980) Fibronectin in early avian embryos: Synthesis and distribution along the migration pathways of neural crest cells. Cell Tissue Res 211, 269-291

Newgreen DF, Gibbins IL, Sauter J, Wallenfels B, Wütz R (1982) Ultrastructural and tissueculture studies on the role of fibronectin, collagen and glycosaminoglycans in the migration of neural crest cells in the fowl embryo. Cell Tissue Res 221, 521-549

Noden DM (1975) An analysis of the migratory behavior of avian cephalic neural crest cells. Dev Biol 42, 106-130

Obara M, Kang MS, Yamada KM (1988) Sitedirected mutagenesis of the cell-binding domain of human fibronectin: Separable, synergistic sites mediate adhesive function. Cell $53,649-657$

Owens RJ, Baralle FE (1986) Mapping the collagen-binding site of human fibronectin by expression in Escherichia coli. EMBO J 5 , 2825-2830

Paul JI, Schwarzbauer JE, Tamkun JW, Hynes RO (1986) Cell-type-specific fibronectin subunits generated by alternative splicing. $J$ Biol Chem 261, 12258-12265

Poole TJ, Thiery JP (1987) Antibodies and a synthetic peptide that block cell-fibronectin adhesion arrest neural crest cell migration in vivo. In: new discoveries and technologies in 
developmental biology (Slavkin HC, ed) Alan R Liss, New York, 235-238

Rickmann M, Fawcett JW, Keynes RJ (1985) The migration of neural crest cells and the growth of motor axons through the rostral half of the chick somite. J Embryol Exp Morphol 90, 437-455

Rovasio RA, Delouvée A, Yamaha KM, Timpl R, Thiery JP (1983) Neural crest cell migration: requirement for exogenous fibronectin and high cell density. J Cell Biol 96, 462-473

Suzuki S, Argraves WS, Pytela R, Arai H, Krusius T, Pierschbacher MD, Ruosiahti E (1986) cDNA and amino-acid sequences of the cell adhesion protein receptor recognizing vitronectin reveals a transmembrane domain and homologies with other adhesion protein receptors. Proc Natl Acad Sci USA 83, 8614-8618

Tamkun JW, DeSimone DW, Fonda D, Patel RS, Buck C, Horwitz AF, Hynes RO (1986) Structure of integrin, a glycoprotein involved in the transmembrane linkage between fibronectin and actin. Cell 46, 271-282

Tan SS, Crossin KL, Hoffman S, Edelman GM (1987) Asymmetric expression in somites of cytotactin and its proteoglycan ligand is correlated with neural crest cell distribution. Proc Natt Acad Sci USA 84, 7977-7981

Teillet AM, Kalcheim C, Le Douarin NM (1987) Formation of the dorsal root glanglia in the avian embryo: segmental origin and migratory behavior of neural crest progenitor cells. Dev Biol 120, 329-347

Thiery JP, Duband JL, Delouvée A (1982) Pathways and mechanism of avian trunk neural crest cell migration and localization. Dev Biol 93, 323-343

Tosney KW (1978) The early migration of neural crest cells in the trunk region of the avian embryo. An electron microscopic study. Dev Biol $62,317-333$

Tosney KW (1982) The segregation and early migration of cranial neural crest cells in the avian embryo. Dev Biol 89, 13-24

Tucker RP, Erickson GA (1984) Morphology and behavior of quail neural crest cells in artificial three dimensional extracellular matrices. Dev Biol 104, 390-405

Tucker RP, Edwards BF, Erickson CA (1985) Tension in the culture dish: microfilament organization and migratory behavior of quail neural crest cells. Cell Motil 5, 225-237

Valinsky JE, Le Douarin NM (1985) Production of plasminogen activator by migrating cephalic neural crest cells. EMBO J 4, 1403-1406

Vincent M, Thiery JP (1984) A cell surface marker for neural crest and placodal cells: further evolution in peripheral and central nervous system. Dev Biol 103, 468-481

Weston JA (1963) A radioautographic analysis of the migration and localization of trunk crest cells in the chick. Dev Biol 6, 279-310

Weston JA (1982) Mobile and social behavior of neural crest cells. In: cell behaviour (Bellairs R, Curtis A, Dunn GA, eds) Cambridge Univ Press, Cambridge, 429-470

Yamada KM, Yamada SS, Pastan I (1976) Cell surface protein partially restores morphology, adhesiveness, and contact inhibition of movement to transformed fibroblasts. Proc Natl Acad Sci USA 73, 1217-1221 